\title{
Growth series for Artin groups of dihedral type
}

\author{
Jean MAIREsse * and Frédéric Mathéus ${ }^{\dagger}$
}

November 2, 2005

\begin{abstract}
We consider the Artin groups of dihedral type $I_{2}(k)$ defined by the presentation $A_{k}=$ $\langle a, b \mid \operatorname{prod}(a, b ; k)=\operatorname{prod}(b, a ; k)\rangle \operatorname{where} \operatorname{prod}(s, t ; k)=s t s t s \ldots$, with $k$ terms in the product on the right-hand side. We prove that the spherical growth series and the geodesic growth series of $A_{k}$ with respect to the Artin generators $\left\{a, b, a^{-1}, b^{-1}\right\}$ are rational. We provide explicit formulas for the series.
\end{abstract}

Keywords: Artin group of dihedral type, spherical growth series, geodesic growth series, automatic group, translation length.

AMS classification (2000): Primary 20F10, 20F36; Secondary 20F69, 68Q45, 68R15.

\section{Introduction}

The spherical and geodesic growth series $\mathcal{S}$ and $\mathcal{G}$ of a finitely-generated group, formally defined in (4) and (5), are a way to capture the combinatorial structure of the group. As such, they are attracting a lot of attention, see for instance [17] and the references therein. In many cases, these series are known to be rational. This is usually proved as follows: for $\mathcal{S}$ by exhibiting a geodesic cross-section which is regular, for $\mathcal{G}$ by showing that the set of all geodesics is regular.

Consider the braid group on three strands $B_{3}=\langle a, b \mid a b a=b a b\rangle$ and the set of Artin generators $S=\left\{a, b, a^{-1}, b^{-1}\right\}$. The pair $\left(B_{3}, S\right)$ is well understood. When run over $\left(B_{3}, S\right)$, the GAP package KBMAG provides a short-lex automatic structure, see [14, Example 6.1]. In particular, there exists a regular geodesic cross-section, and, therefore, $\mathcal{S}$ is rational. The explicit rational expression for $\mathcal{S}$ is given in [25, Theorem 5.3]. The regularity of the set of all geodesics is proved in Sabalka [25], and an explicit formula for $\mathcal{G}$ is derived. The proof is based on a careful study of the structure of the Cayley graph.

The extension to other braid groups is an intriguing open problem, mentioned for instance in [19] or [10]. That is, consider the braid group on $n \geqslant 4$ strands $B_{n}=\left\langle a_{1}, \ldots, a_{n-1}\right|$ $\left.\forall i, a_{i} a_{i+1} a_{i}=a_{i+1} a_{i} a_{i+1}, \quad \forall i, j,|j-1|>1, a_{i} a_{j}=a_{j} a_{i}\right\rangle$ and the Artin generators $S=$ $\left\{a_{1}, \ldots, a_{n-1}, a_{1}^{-1}, \ldots, a_{n-1}^{-1}\right\}$. Is the set of all geodesics of $\left(B_{n}, S\right)$ regular? Does there exist a geodesic cross-section which is regular?

The same two questions can be asked for any pair formed by an Artin group of finite Coxeter type and its Artin generators. Among these groups, there are two natural families generalizing

\footnotetext{
*LIAFA, CNRS-Université Paris 7, case 7014, 2, place Jussieu, 75251 Paris Cedex 05, France. E-mail: Jean.Mairesse@liafa.jussieu.fr

${ }^{\dagger}$ LMAM, Université de Bretagne-Sud, Campus de Tohannic, BP 573, 56017 Vannes, France. E-mail: Frederic.Matheus@univ-ubs.fr
} 
$B_{3}$ : the braid groups and the Artin groups of dihedral type $I_{2}(n)$. In this paper, we answer the above two questions positively for Artin groups of dihedral type.

Set $\operatorname{prod}(u, v ; k)=u v u \cdots$, with $k$ letters on the right-hand side. For $n \geqslant 3$, consider the Artin group $A_{n}$ of dihedral type $I_{2}(n)$ and the set of Artin generators $S$ defined by:

$$
A_{n}=\langle a, b \mid \operatorname{prod}(a, b ; n)=\operatorname{prod}(b, a ; n)\rangle, \quad S=\left\{a, b, a^{-1}, b^{-1}\right\} .
$$

Observe that $A_{3}=B_{3}$. We prove that the pair $\left(A_{n}, S\right)$ enjoys the same properties as the pair $\left(B_{3}, S\right)$. We exhibit a finite automaton recognizing the set of all geodesics, and a finite automaton recognizing a specific geodesic cross-section. The explicit formulas for $\mathcal{G}$ and $\mathcal{S}$ are given in Theorem 3.1. When specializing to $n=3$, we recover the above mentionned results with shorter proofs.

The Artin generators of $A_{n}$ or $B_{n}$ are natural in several respect. First, they are minimal for inclusion. Second, they correspond to natural generators in the associated Coxeter group. Third, in $B_{n}$, they have a nice interpretation in terms of braids. However, they are more complicated to deal with than other a priori less natural sets of generators. More specifically, let $T$ be the set of Garside generators associated with the Garside normal form [15, 13, 8] (several variants are possible for $T$ ). Typically, for $A_{n}$, one may consider

$$
T=\{a, b, a b, b a, \cdots, \operatorname{prod}(a, b ; n-1), \operatorname{prod}(b, a ; n-1), \operatorname{prod}(a, b ; n)=\operatorname{prod}(b, a ; n)\}^{ \pm} .
$$

Then the growth series with respect to $T$ are rational. Most of the results hold not only for $A_{n}$ or $B_{n}$ but more generally for Artin groups of finite Coxeter type. This approach has been pursued by Charney and others. For precise statements, see [5, 8, 9, 10].

The generators in (2) have different lengths with respect to $S$. Hence, geodesics with respect to $S$ and $T$ may be very different. And knowing the growth series with respect to $T$ does not help in getting the growth series with respect to $S$. Our study also starts with the Garside normal form. But then we need an additional ingredient which is a procedure to go from a Garside geodesic to an Artin geodesic. Such a procedure was proposed by Berger [2] for $B_{3}$ and we extend it to $A_{n}$. Here we use it to prove a simple characterization of geodesics (Proposition 4.3). Eventually the growth series for $S$ that we obtain in Theorem 3.1 have a more complicated structure than their counterpart for $T$ derived in $[9,10]$ and recalled in (19)-(20).

In the monoid case, the situation is simpler. Indeed, dealing with Garside or Artin generators is virtually the same for the following reason. View the left-hand side of (1) as a monoid presentation. This defines the positive Artin monoid $A_{n}^{+}$. The positive braid monoid $B_{n}^{+}$is defined similarly. The relations defining $A_{n}^{+}$or $B_{n}^{+}$are equal-length relations with respect to the Artin generators $S^{+}$(for $\left.A_{n}^{+}, S^{+}=\{a, b\}\right)$. It follows that all the words over $S^{+}$are geodesic. Now consider the set of Garside normal forms. For the same reason, it provides a geodesic cross-section not only over the Garside generators $T^{+}$but also over the Artin generators $S^{+}$. The approach extends to all Artin monoids of finite Coxeter type for which the spherical growth series are therefore computable. Explicit computations have been carried out in $[6,28]$.

The group $A_{n}$ admits the following so-called dual presentation: $\widehat{A}_{n}=\left\langle\sigma_{1}, \ldots, \sigma_{n}\right| \sigma_{1} \sigma_{2}=$ $\left.\sigma_{2} \sigma_{3}=\cdots=\sigma_{n} \sigma_{1}\right\rangle$. Let $\widehat{S}=\left\{\sigma_{1}, \ldots, \sigma_{n}\right\}^{ \pm}$be the corresponding set of dual generators. One may associate with this presentation a dual Garside-type normal form. And this does not require a change of generators, in other words $\widehat{S}$ plays the role of both $S$ and $T$. Consequently, the generating series of $\left(\widehat{A}_{n}, \widehat{S}\right)$ can be computed using an approach similar to the one used for $\left(A_{n}, T\right)$. For instance, the spherical growth series of $\widehat{A}_{3}$ is computed in [27, Theorem 9], and the geodesic growth series of $\widehat{A}_{n}$ is given in [10, Example 4.3]. 
Now let us consider the braid groups $B_{n}, n \geqslant 4$. They also admit a dual presentation $\widehat{B}_{n}$ and dual generators $\widehat{S}$, introduced by Birman, Ko, and Lee [4].

Now, working with the Garside-type normal form of $\widehat{B}_{n}$ requires a switch to a new set of generators $\widehat{T} \neq \widehat{S}$. And the elements of $\widehat{T}$ have different lengths with respect to $\widehat{S}$. Consequently, the situation is as follows. On the one hand, computing the generating series of $\left(\widehat{B}_{n}, \widehat{T}\right)$, à la Charney, is feasible (see [10, Corollary 4.2]). On the other hand, computing the generating series of $\left(\widehat{B}_{n}, \widehat{S}\right)$ is presumably as difficult as computing the generating series of $\left(B_{n}, S\right)$. In particular, we can state the following dual open problem. Is the set of all geodesics of $\left(\widehat{B}_{n}, \widehat{S}\right)$ regular? Does there exist a geodesic cross-section which is regular?

The same two questions can again be asked for any Artin group of finite Coxeter type. Indeed, a dual structure may be defined for all such groups. This is linked to Garside monoids and is a subject of active research, see for instance [3, 4, 11, 24].

\section{Preliminaries}

\subsection{Growth series}

Given a finite set $\Sigma$, the free monoid it generates is denoted by $\Sigma^{*}$. The length (number of letters) of a word $w$ is denoted by $|w|_{\Sigma}$. We denote by $\operatorname{Rat}\left(\Sigma^{*}\right)$, the set of regular languages of $\Sigma^{*}$, that is the set of languages associated with rational expressions of $\Sigma^{*}$, or equivalently (Kleene Theorem) the set of languages recognized by a finite automaton. The counting series $\left(\sum_{u \in L} x^{|u|_{\Sigma}}\right)$ associated with a regular language $L$ is a rational series of $\mathbb{N}[[x]]$.

Let $(G, *)$ be a finitely generated group. Let $\Sigma \subset G$ be a finite set of generators of $G$ with $1_{G} \notin \Sigma$ and $s \in \Sigma \Longrightarrow s^{-1} \in \Sigma$. Denote by $\pi: \Sigma^{*} \rightarrow G$ the monoid homomorphism which associates to a word $a_{1} \cdots a_{k}$ the group element $a_{1} * \cdots * a_{k}$. A word $w \in \pi^{-1}(g)$ is called a representative of $g$. The length with respect to $\Sigma$ of a group element $g$ is:

$$
|g|_{\Sigma}=\min \left\{k \mid g=s_{1} * \cdots * s_{k}, s_{i} \in \Sigma\right\} .
$$

A representative $w$ of $g$ is a geodesic (word) if $|w|_{\Sigma}=|g|_{\Sigma}$.

A language $L$ of $\Sigma^{*}$ is a cross-section of $G$ (over the alphabet $\Sigma$ ) if the restriction of $\pi$ to $L$ defines a bijection, that is if every element of $G$ has a unique representative in $L$. A word of $L$ is then called a normal form word.

The spherical growth series of $G$ with respect to $\Sigma$ is the formal series $\mathcal{S}(G, \Sigma) \in \mathbb{N}[[x]]$ defined by:

$$
\mathcal{S}(G, \Sigma)=\sum_{g \in G} x^{|g|_{\Sigma}}=\sum_{n \in \mathbb{N}} \#\left\{\left.g \in G|| g\right|_{\Sigma}=n\right\} x^{n}
$$

The geodesic growth series of $G$ with respect to $\Sigma$ is the series $\mathcal{G}(G, \Sigma) \in \mathbb{N}[[x]]$ defined by:

$$
\mathcal{G}(G, \Sigma)=\sum_{g \in G} \#\left\{\left.u \in \pi^{-1}(g)|| u\right|_{\Sigma}=|g|_{\Sigma}\right\} x^{|g|_{\Sigma}}=\sum_{n \in \mathbb{N}} \#\left\{\left.w \in \Sigma^{*}|| w\right|_{\Sigma}=|\pi(w)|_{\Sigma}=n\right\} x^{n}
$$

If the set of all the geodesics is regular, the pair $(G, \Sigma)$ is said to be strongly geodesic regular, or to form a Cannon pair [21]. It implies that $\mathcal{G}$ is rational. If there exists a geodesic cross-section, the pair is unique geodesic regular and it implies that $\mathcal{S}$ is rational.

The two notions of strong and unique geodesic regularity depend on the pair group-generators and not on the group alone, see for instance [13, Example 4.4.2] and [26]. On the other hand, a word-hyperbolic group with any finite set of generators is strongly and unique geodesic regular. 
More precisely, the following diagram of implications holds (for definitions and background, see $[18,16]$ on word-hyperbolic groups and $[13]$ on automatic groups):

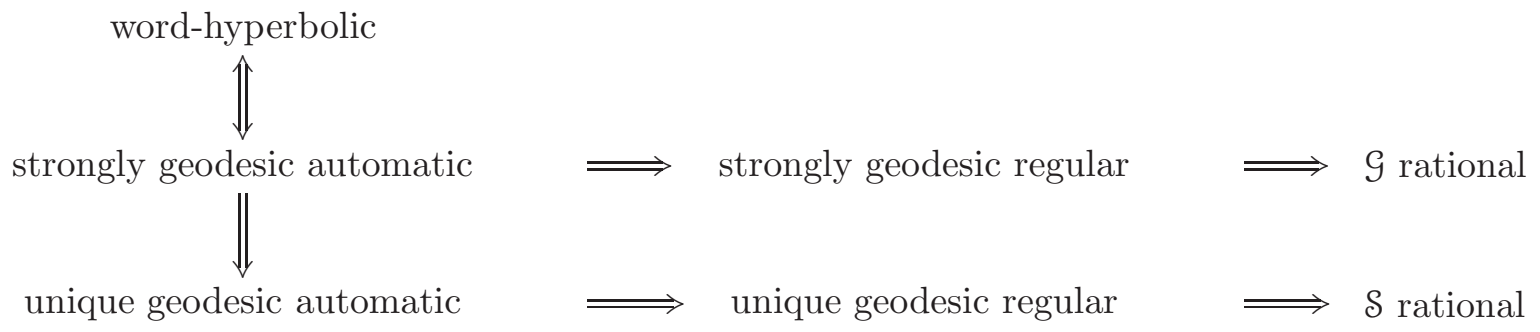

The implication [strongly geodesic automatic] $\Longrightarrow$ [unique geodesic automatic] is proved in [13, Corollary 2.5.2]. The implication [word-hyperbolic] $\Longrightarrow$ [strongly geodesic automatic] is essentially due to Cannon [7], and the converse is due to Papasoglu [23]. All the other implications are immediate consequences of the definitions.

Below we consider the family $\left(A_{k}\right)_{k \geqslant 3}$ of Artin groups of dihedral type, together with the Artin generators $S$. The groups $A_{k}$ are not word-hyperbolic. We prove that $\left(A_{k}, S\right)$ is strongly geodesic regular and unique geodesic regular. Very roughly, the spirit of the proof is as follows. First, the quotient $A_{k} / Z$, where $Z$ is the center of $A_{k}$, is word-hyperbolic. So we can deal with $\left(A_{k} / Z, S\right)$. Second, there is a way to go from the geodesics in $A_{k} / Z$ to the geodesics in $A_{k}$.

\subsection{Artin groups of dihedral type}

For $k \geqslant 3$, we denote by $A_{k}$ the Artin group of dihedral type $I_{2}(k)$. This group is defined by the (group) presentation:

$$
A_{k}=\langle a, b \mid \operatorname{prod}(a, b ; k)=\operatorname{prod}(b, a ; k)\rangle,
$$

where $\operatorname{prod}(s, t ; k)=$ ststs..., with $k$ terms in the product on the right-hand side. Observe that $A_{3}=B_{3}$, the braid group on three strands. The Coxeter group of $A_{k}$ is: $W_{k}=\langle a, b| a^{2}=b^{2}=$ $\left.(a b)^{k}=1\right\rangle$. The group $W_{k}$ is the dihedral group of order $2 k$, hence the name of $A_{k}$. Set

$$
\Delta=\operatorname{prod}(a, b ; k)=\operatorname{prod}(b, a ; k) .
$$

The center $Z$ of $A_{k}$ is generated by $\Delta^{2}$ if $k$ is odd, and by $\Delta$ if $k$ is even, see for instance [22].

Let $A_{k}^{+}$be the monoid defined by (6) interpreted as a monoid presentation. The Artin generators of $A_{k}$ and $A_{k}^{+}$are respectively:

$$
S=\left\{a, b, a^{-1}, b^{-1}\right\}, \quad S^{+}=\{a, b\} .
$$

Denote by $\pi: S^{*} \rightarrow A_{k}$ the canonical morphism.

Lemma 2.1. The monoid $A_{k}^{+}$injects into the group $A_{k}$.

See [12], or [22, Cor. 3.2] for a proof. It is therefore licit to view $A_{k}^{+}$as a subset of $A_{k}$. Besides, observe that all the relations defining $A_{k}^{+}$are equal-length relations (with respect to $S^{+}$). A consequence of Lemma 2.1 is then:

$$
\forall u \in\left(S^{+}\right)^{*}, \quad|u|_{S^{+}}=|\pi(u)|_{S} .
$$

That is, all the words containing only the letters $a$ and $b$ are geodesic words of $A_{k}$. Similarly, all the words containing only $a^{-1}$ and $b^{-1}$ are geodesic. 
A central ingredient in what follows is a normal form due to Garside [15] (see also $[12,13,8]$ ). It is defined for all Artin groups of finite Coxeter type. For dihedral Artin groups, the statement is the following. Set $M^{+}=\{a, b, a b, b a, \ldots, \operatorname{prod}(a, b ; k-1), \operatorname{prod}(b, a ; k-1)\}$. For $u \in M^{+}$, let $\operatorname{First}(u) \in S^{+}$be the first letter of $u$, and let Last $(u) \in S^{+}$be the last letter of $u$.

Proposition 2.2. For every $g \in A_{k}$, one can find $\delta \in \mathbb{Z}$ and $g_{1}, \ldots, g_{m} \in M^{+}$such that

$$
g=g_{1} \cdot \ldots \cdot g_{m} \cdot \Delta^{\delta} .
$$

If $m$ is minimal, such a decomposition is unique. We call the decomposition with $m$ minimal the Garside normal form of $g$. Moreover, $g_{1} \cdot \ldots \cdot g_{m} \cdot \Delta^{\delta}, g_{i} \in M^{+}$, is the Garside normal form of an element in $A_{k}$ if and only if:

$$
\forall i \in\{1, \ldots, m-1\}, \operatorname{Last}\left(g_{i}\right)=\operatorname{First}\left(g_{i+1}\right) .
$$

Formally, a Garside normal form is an element of the monoid $\left(M^{+} \cup\left\{\Delta, \Delta^{-1}\right\}\right)^{*}$. The condition in (10) implies that the set of Garside normal forms is a regular language of $\left(M^{+} \cup\left\{\Delta, \Delta^{-1}\right\}\right)^{*}$. The Garside normal form $g_{1} \cdot \ldots \cdot g_{m} \cdot \Delta^{\delta}$ of $g$, viewed as an element of $S^{*}$, is a geodesic representative of $g$ with respect to $S$ if and only if $\delta \geqslant 0$. For the if direction, use (8). For the only if direction, observe that, for all $i \in\{1, \ldots, k-1\}$ :

$$
\begin{gathered}
\pi\left(\operatorname{prod}(a, b ; i) \Delta^{-1}\right)=\pi\left(\operatorname{prod}\left(b^{-1}, a^{-1} ; k-i\right)\right) \\
\left|\operatorname{prod}(a, b ; i) \Delta^{-1}\right|_{S}=k+i, \quad\left|\operatorname{prod}\left(b^{-1}, a^{-1} ; k-i\right)\right|_{S}=k-i .
\end{gathered}
$$

Let us introduce two more notations. Following $[8,9]$, we write

$$
\bar{g}=\Delta g \Delta^{-1}=\Delta^{-1} g \Delta
$$

(since $\Delta^{2}$ is central) for the conjugate of $g$ by $\Delta$. For instance, if $k$ is odd, one has $\bar{a}=b$ and $\bar{b}=a$. If $k$ is even, $\Delta$ is central so $\bar{a}=a$ and $\bar{b}=b$. Next, for $g \in M^{+}$, we set

$$
g^{*}=\Delta g^{-1} .
$$

Note that $g^{*} \in M^{+}$, and that $\overline{g^{*}}=\bar{g}^{*}=\bar{g}^{-1} \Delta$. Keeping in mind that $g^{*} \cdot g=\Delta$, one may think of $g^{*}$ as the left-complement of $g$ to get $\Delta$. For instance, in $A_{3}=B_{3}$, we have $a^{*}=a b, b^{*}=b a,(a b)^{*}=b$, and $(b a)^{*}=a$.

Consider the following algorithm.

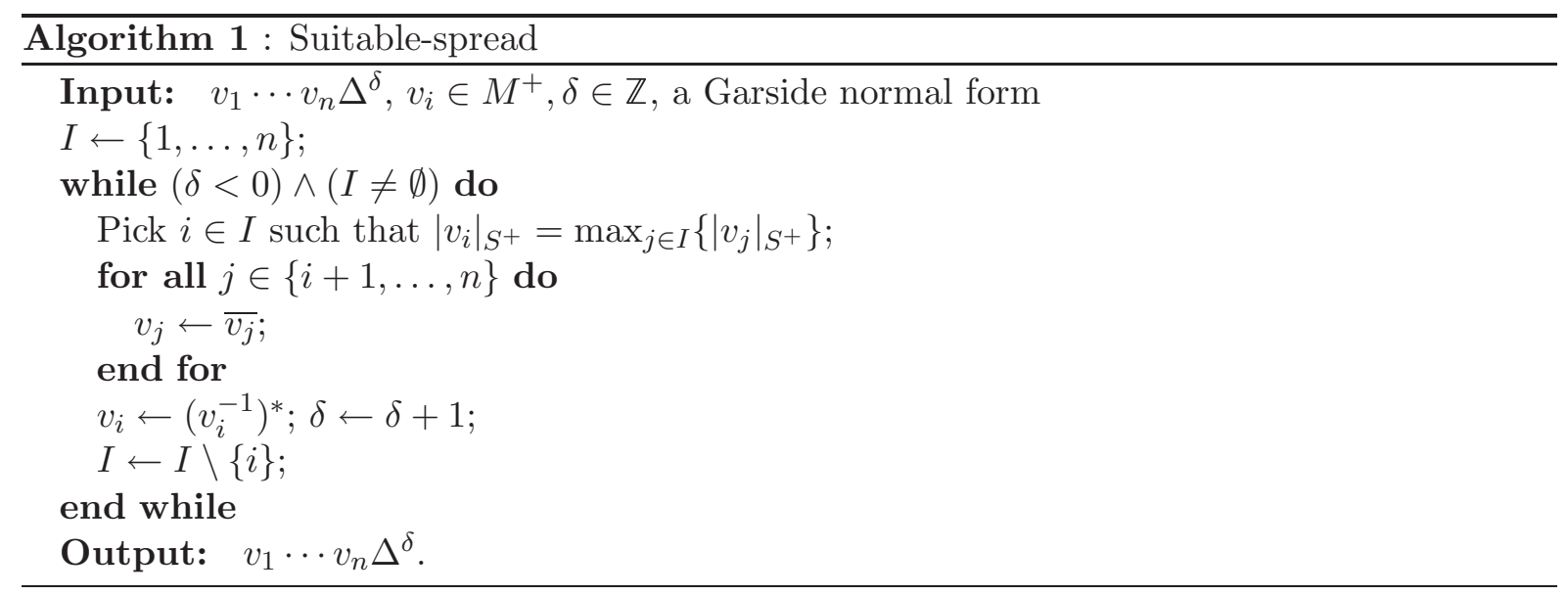


The input and the output of the SUITABLE-SPREAD procedure are clearly two representatives of the same group element. Observe that, according to the choices made in the "Pick $i$ " line, there may be several different outputs for a given input. Observe also that any output $v_{1} \cdots v_{n} \Delta^{\delta}$ satisfies:

$$
[\delta>0] \Longrightarrow\left[\forall i, v_{i} \in M^{+}\right], \quad[\delta<0] \Longrightarrow\left[\forall i, v_{i} \in M^{-}\right], \quad \forall i, \operatorname{Last}\left(v_{i}\right)=\operatorname{First}\left(v_{i+1}\right)
$$

where the definition of First (.) and Last(.) on $M^{-}$is given in (21). We have the following result.

Proposition 2.3. The SuITABlE-SPREAD procedure applied to the Garside normal form of an element $g \in A_{k}$ leads to a representative of $g$ which is geodesic with respect to $S$.

For $A_{3}=B_{3}$, this is proved in [2]. We now prove the result for $A_{k}$ with a different type of argument.

Proof. Consider a group element $g$ with Garside normal form $v=v_{1} \cdots v_{m} \Delta^{\delta}, v_{i} \in M^{+}, \delta \in \mathbb{Z}$. Assume first that $\delta \geq 0$. The output from the SuITABLE-SPREAD procedure is the input word itself. Now, according to Lemma 2.1 and Eq. 8, the word $v$ is indeed of minimal length with respect to $S$.

Next, assume that $\delta \leq-m$. In the SuitaBle-SPREAD procedure, all the $v_{i}$ 's are going to be picked and transformed. Therefore, the output is

$$
w=\left(\tilde{v}_{1} \Delta^{-1}\right) \cdots\left(\tilde{v}_{m} \Delta^{-1}\right) \cdot \Delta^{\delta+m}
$$

where $\tilde{v}_{j}=v_{j}$ if $j$ is odd and $\tilde{v}_{j}=\bar{v}_{j}$ if $j$ is even. Observe that $w$, viewed as an element of $S^{*}$, contains only the letters $\left\{a^{-1}, b^{-1}\right\}$. Using the observation following (8), the word $w$ is therefore geodesic with respect to $S$.

Finally, suppose that $-m<\delta<0$. Among all the ways of spreading the inverses of $\Delta$ among $v_{1}, \ldots, v_{m}$, and doing the transformations $\left[v_{i} \Delta^{-1} \rightarrow\left(v_{i}^{*}\right)^{-1}\right]$, it is clear that the way that leads to the smallest length consists in choosing $|\delta|$ elements $v_{i}$ of maximal possible length. What remains to be proved is that this choice leads to a word whose length is actually $|g|_{S}$.

Let $w$ be a word of minimal length representing $g$. There is a unique decomposition of $w$ as $w=w_{1} \cdot w_{2} \cdots w_{\ell}$, where the words $w_{i}$ are non-empty and built alternatively from the alphabets $\{a, b\}$ and $\left\{a^{-1}, b^{-1}\right\}$. We carry out the proof for $w=w_{1} \cdot w_{2}$ with $w_{1} \in\left\{a^{-1}, b^{-1}\right\}^{*}$ and $w_{2} \in\{a, b\}^{*}$. The proof for the general case follows easily. Let $x_{1}=u_{1} \cdots u_{m_{1}} \cdot \Delta^{\delta_{1}}$ be the Garside normal form of (the element represented by) $w_{1}$. Let $x_{2}=v_{1} \cdots v_{m_{2}} \cdot \Delta^{\delta_{2}}$ be the Garside normal form of $w_{2}$. We first observe that we must have $\delta_{1}=-m_{1}$ and $\delta_{2}=0$. Otherwise, we would contradict the minimality of $w$. (For a detailed argument, see the proof of Proposition 4.3 , case $I+J>k$.) Assume for simplicity that $m_{1}$ is even (the odd case is treated similarly). With this choice of parity, we have

$$
w_{1}=\left(u_{1} \Delta^{-1}\right) \cdots\left(\bar{u}_{m_{1}} \Delta^{-1}\right), \quad w_{2}=v_{1} \cdots v_{m_{2}} .
$$

We claim that the Garside normal form of $g$ is $z=u_{1} \cdots u_{m_{1}} \cdot v_{1} \cdots v_{m_{2}} \cdot \Delta^{-m_{1}}$. According to the local characterization in (10), it is enough to prove that Last $\left(u_{m_{1}}\right)=\operatorname{First}\left(v_{1}\right)$. Assume it is not the case, with for instance Last $\left(u_{m_{1}}\right)=b$ and First $\left(v_{1}\right)=a$. Then the last letter in $w_{1}$, which is the last letter in $\left(\bar{u}_{m_{1}} \Delta^{-1}\right)$, is actually $a^{-1}$. We conclude that the last letter of $w_{1}$ is the inverse of the first letter of $w_{2}$, which contradicts the fact that $w$ is minimal.

Now, starting from $z$, there is obviously a way of spreading the $\Delta^{-1}$ to get $w$. This completes the proof. 


\section{Statement of the results}

For $n \in \mathbb{N}$, let $X_{n} \in \mathbb{N}[x]$ be defined by: $X_{0}=0$ and, $\forall n \geqslant 1$,

$$
X_{n}=x+x^{2}+\cdots+x^{n} .
$$

Theorem 3.1. Consider the Artin group $A_{k}$ of dihedral type $I_{2}(k)$ with the Artin generators $S$. The set of all the geodesics is regular, and there exists a geodesic cross-section which is regular. The geodesic and spherical growth series are:

$$
\begin{gathered}
\mathcal{G}\left(A_{k}, S\right)=\sum_{\substack{I, J \geqslant 1 \\
I+J=k}} \frac{1+X_{I}+X_{J}}{1-X_{I}-X_{J}}-\sum_{\substack{I, J \geqslant 1 \\
I+J=k-1}} \frac{1+X_{I}+X_{J}}{1-X_{I}-X_{J}}+2 \frac{1}{1-2 x}-2 \frac{1+X_{k-1}}{1-X_{k-1}} \\
\mathcal{S}\left(A_{k}, S\right)=\sum_{\substack{I, J \geqslant 1 \\
I+J=k-1}} \frac{1+X_{I}+X_{J}}{1-X_{I}-X_{J}}-\sum_{\substack{I, J \geqslant 1 \\
I+J=k-2}} \frac{1+X_{I}+X_{J}}{1-X_{I}-X_{J}}+2 \frac{1+X_{k-1}}{1-X_{k-1}} \frac{1}{1-x^{k}} \\
-2 \frac{1+X_{k-2}}{1-X_{k-2}}+\sum_{\substack{I, J \geqslant 1 \\
I+J=k}} \frac{2 x^{k}}{\left(1-X_{I-1}-X_{J-1}\right)\left(1-X_{I-1}-X_{J}\right)\left(1-X_{I}-X_{J-1}\right)}
\end{gathered}
$$

We also give an explicit description of: (i) an automaton recognizing the set of all the geodesics; (ii) an automaton recognizing a geodesic cross-section. The expressions in (15) and (16) are byproducts of this description.

For $A_{3}$ and $A_{4}$, we have:

$$
\begin{aligned}
& \mathcal{G}\left(A_{3}, S\right)=1+\frac{4 x}{\left(1-x-x^{2}\right)\left(1-2 x-x^{2}\right)} \\
& \mathcal{S}\left(A_{3}, S\right)=1+\frac{2 x\left(2-2 x-x^{2}\right)}{(1-x)(1-2 x)\left(1-x-x^{2}\right)} \\
& \mathcal{G}\left(A_{4}, S\right)=1+\frac{4 x(1-x)(1+x)\left(1-6 x+10 x^{2}-x^{3}-3 x^{4}-5 x^{5}-2 x^{6}-x^{7}\right)}{(1-2 x)\left(1-2 x-x^{2}\right)\left(1-2 x-2 x^{2}\right)\left(1-x-x^{2}-x^{3}\right)\left(1-2 x-x^{2}-x^{3}\right)} \\
& \mathcal{S}\left(A_{4}, S\right)=1+\frac{4 x\left(1-x-x^{2}\right)\left(1-2 x-x^{3}\right)}{(1-x)\left(1-x-x^{2}-x^{3}\right)\left(1-2 x-x^{2}\right)^{2}} .
\end{aligned}
$$

For instance, $\mathcal{G}\left(A_{4}, S\right)=1+4 x+12 x^{2}+36 x^{3}+108 x^{4}+308 x^{5}+868 x^{6}+2420 x^{7}+O\left(x^{8}\right)$ and $\mathcal{S}\left(A_{4}, S\right)=1+4 x+12 x^{2}+36 x^{3}+100 x^{4}+268 x^{5}+708 x^{6}+1848 x^{7}+O\left(x^{8}\right)$. The formulas for $A_{3}$ appear in [25], see the discussion in the Introduction.

Formula (16) enables to compute the growth rate of the volume of the spheres in the Cayley graph of $\left(A_{k}, S\right)$. One has:

$$
\lim _{n \rightarrow \infty} \frac{1}{n} \log \#\left\{\left.g \in A_{k}|| g\right|_{S}=n\right\}=\log \left(\rho_{k}\right),
$$

where $\rho_{3}=2$, and where, for $k \geq 4, \rho_{k}$ is the inverse of the minimal module of a root of $1-2 x-x^{2}-\cdots-x^{k-2}$. For instance, $\rho_{4}=\sqrt{2}+1$.

For comparison, consider

$$
T=\{a, b, a b, b a, \cdots, \operatorname{prod}(a, b ; k-1), \operatorname{prod}(b, a ; k-1), \Delta\}^{ \pm} .
$$


We have:

$$
\begin{aligned}
& \mathcal{G}\left(A_{k}, T\right)=1+\frac{2 x\left[(k-1)\left(2 k^{2}-3 k+4\right) x^{2}-(2 k-1)^{2} x+2 k-1\right]}{(1-x)(1-(k-1) x)(1-k x)(1-2(k-1) x)} \\
& \mathcal{S}\left(A_{k}, T\right)=1+\frac{2 x[1+(k-1)(2-2 x-(k-1) x)]}{(1-x)(1-(k-1) x)^{2}} .
\end{aligned}
$$

The formula for $\mathcal{S}\left(A_{k}, T\right)$ appears in [9, Example 4.5] and the one for $\mathcal{G}\left(A_{k}, T\right)$ is given in [10, Example 4.4]. The combinatorial structure of $\left(A_{k}, S\right)$ is more complex than the one of $\left(A_{k}, T\right)$. This is reflected in the respective number of poles of the series in (15)-(16) and (19)-(20).

\section{Geodesic words}

The goal is to provide an algorithm to decide if a word is geodesic in $A_{k}$ with respect to $S$.

We first introduce a couple of notations. Denote by $\mathbb{E}$ the group freely generated by $S^{+}$. Any element $u \in \mathbb{F}$ is uniquely represented by a reduced word of $S^{*}$, that is a word $u_{1} \cdot \ldots \cdot u_{l} \in S^{*}$ such that $u_{i} \neq u_{i+1}^{-1}$ for all $i \in\{1, \ldots, l-1\}$. Below, we identify an element of $\mathbb{E}$ with the corresponding reduced word of $S^{*}$. We still denote by $\pi: \mathbb{F} \rightarrow A_{k}$ the canonical homomorphism and we still say that $u$ is a representative of $g$ if $\pi(u)=g$.

For all $n \geqslant 1$, set $\Sigma_{n}=\Sigma_{n}^{+} \sqcup \Sigma_{n}^{-}$with

$$
\begin{aligned}
& \Sigma_{n}^{+}=\{\operatorname{prod}(a, b ; j), \operatorname{prod}(b, a ; j) ; 1 \leqslant j \leqslant n\} \\
& \Sigma_{n}^{-}=\left\{\operatorname{prod}\left(a^{-1}, b^{-1} ; j\right), \operatorname{prod}\left(b^{-1}, a^{-1} ; j\right) ; 1 \leqslant j \leqslant n\right\} .
\end{aligned}
$$

Set also

$$
M=\Sigma_{k-1}, \quad M^{+}=\Sigma_{k-1}^{+}, \quad M^{-}=\Sigma_{k-1}^{-}
$$

At last, set

$$
T=\Sigma_{k-1} \cup\left\{\Delta, \Delta^{-1}\right\}, \quad T^{+}=\Sigma_{k-1}^{+} \cup\{\Delta\}, \quad T^{-}=\Sigma_{k-1}^{-} \cup\left\{\Delta^{-1}\right\} .
$$

Observe that $T$ is the set of generators in (18). For instance, for $k=3$, one has:

$$
\Sigma_{3}^{+}=\{a, b, a b, b a, a b a, b a b\}, \quad M^{+}=\{a, b, a b, b a\}, \quad T^{+}=\{a, b, a b, b a, \Delta\} .
$$

Recall that, for $u \in M^{+}$, we defined First $(u) \in S^{+}$as being the first letter of $u$, and Last $(u) \in S^{+}$ as being the last letter of $u$. We now define First(.) and Last(.) for elements of $M^{-}$in the following way (the reason for this weird definition will appear soon):

$$
\operatorname{First}\left(a^{-1} u\right)=b, \quad \operatorname{First}\left(b^{-1} u\right)=a, \quad \operatorname{Last}\left(u a^{-1}\right)=b, \quad \text { and } \quad \operatorname{Last}\left(u b^{-1}\right)=a .
$$

Consider the algorithm Bracketting-With-RespeCt-TO-T. For $u \in \mathbb{F}$, let $\operatorname{Brack}(u)$ denote the output of the algorithm when applied to the input $u$. Consider $u \in \mathbb{F}$ with $\operatorname{Brack}(u)=v=$ $v_{1} \cdot \ldots \cdot v_{\ell}$. Define

$$
\begin{aligned}
& \operatorname{Pos}(u)=\max \left\{n \mid \exists i, v_{i}=\operatorname{prod}(a, b ; n) \text { or } \operatorname{prod}(b, a ; n)\right\} \\
& \operatorname{Neg}(u)=\max \left\{n \mid \exists i, v_{i}=\operatorname{prod}\left(a^{-1}, b^{-1} ; n\right) \operatorname{or} \operatorname{prod}\left(b^{-1}, a^{-1} ; n\right)\right\}
\end{aligned}
$$

For convenience reasons, we also set $\operatorname{Pos}(v)=\operatorname{Pos}(u)$ and $\operatorname{Neg}(v)=\operatorname{Neg}(u)$. Note that $\operatorname{Pos}(\cdot)$ and $\operatorname{Neg}(\cdot)$ are less than or equal to $k$. 


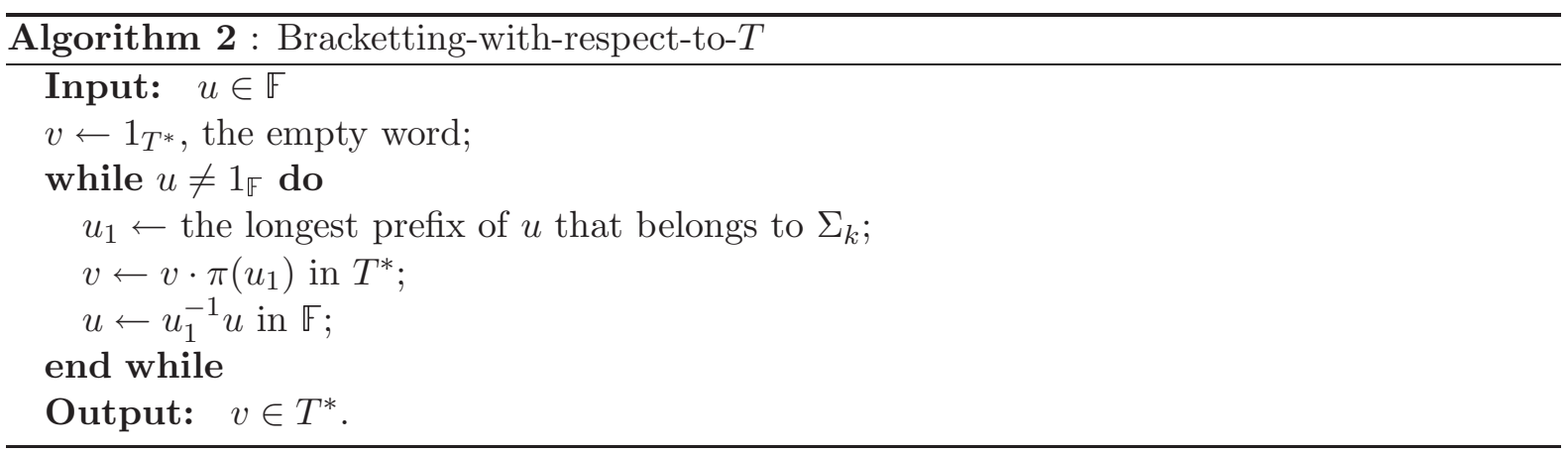

Lemma 4.1. Consider $u \in \mathbb{F}$ with $\operatorname{Brack}(u)=v_{1} \cdot \ldots \cdot v_{\ell}$. If $\operatorname{Pos}(u)<k$ and $N e g(u)<k$ then $\forall i=1, \ldots, \ell-1$,

$$
\operatorname{Last}\left(v_{i}\right)=\operatorname{First}\left(v_{i+1}\right) .
$$

The proof of the Lemma follows directly from the structure of the algorithm. When $\operatorname{Pos}(u)=k$ or $\operatorname{Neg}(u)=k$ then $(23)$ does not make too much sense since we have not defined First $(\cdot)$ and Last $(\cdot)$ for $\Delta$ or $\Delta^{-1}$.

When $\operatorname{Pos}(u)<k$ and $\operatorname{Neg}(u)<k$, one easily obtains the Garside normal form starting from $\operatorname{Brack}(u)$.

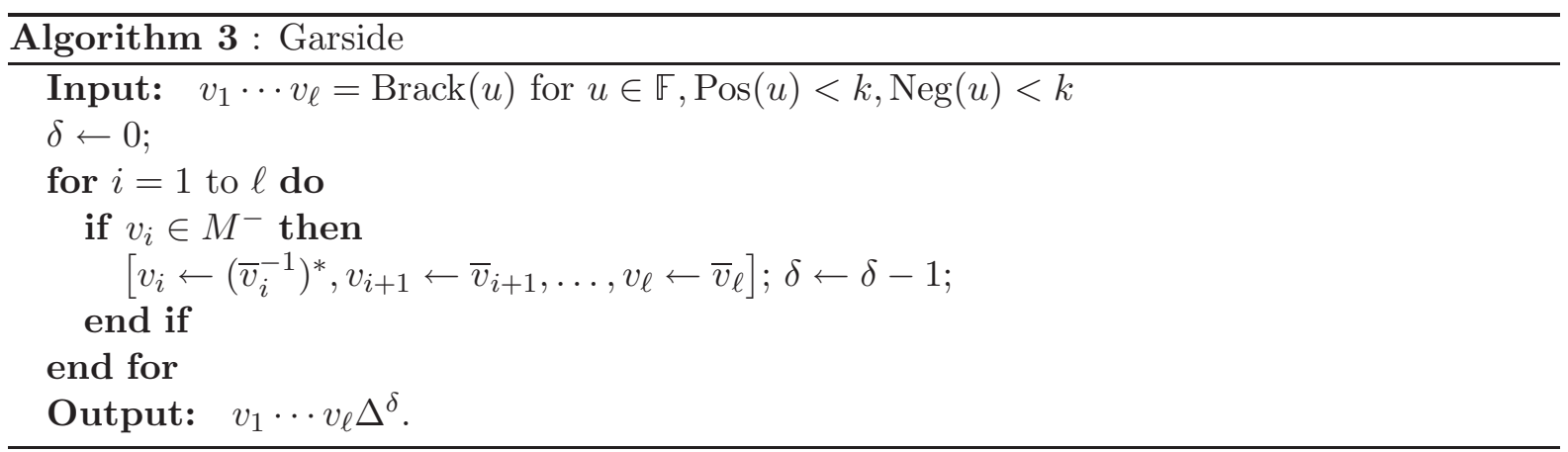

Consider the GaRside algorithm. Denote by $\operatorname{Gars}(v)$ the output of the procedure when applied to the input $v=\operatorname{Brack}(u)$.

Lemma 4.2. Consider $u \in \mathbb{F}$ with $\operatorname{Pos}(u)<k$ and $\operatorname{Neg}(u)<k$. Then $u$, Brack $(u)$, and Gars $\circ$ Brack $(u)$ are representatives of the same group element $g$. Moreover, Gars $\circ$ Brack $(u)$ is the Garside normal form of $g$.

Proof. The argument is in three steps: (a) the input word $v$ satisfies (23); (b) the transformations performed in the GARSIDE algorithm preserve the property Last $\left(u_{i}\right)=\operatorname{First}\left(u_{i+1}\right)$; (c) in the output word, all the elements $v_{i}$ belong to $M^{+}$. Hence $\operatorname{Gars}(v)$ satisfies (10) and is therefore in Garside normal form.

When $\operatorname{Pos}(u)=k$ or $\operatorname{Neg}(u)=k$, then it becomes more subtle to obtain the Garside normal form, and the above algorithm cannot be directly adapted. Consider for instance a group element $g \in A_{3}$ having a representative $u=b b b a b a^{-1} b \in \mathbb{F}$. We have $\operatorname{Brack}(u)=b \cdot b \cdot \Delta \cdot a^{-1} \cdot b$ and $\operatorname{Pos}(u)=3, \operatorname{Neg}(u)=1$. Pushing the $\Delta$ to the right, we get $b b b^{-1} a \Delta$. After simplification, we obtain the Garside normal form of $g$ which is simply $(b a) \cdot \Delta$.

We do not give the algorithm to get the Garside normal form starting from any representative $u \in \mathbb{E}$ since it will not be needed in the sequel. 
Next proposition characterizes the geodesic representatives of the elements of $A_{k}$ with respect to $S$.

Proposition 4.3. Let $g \in A_{k}$, let $u \in \mathbb{F}$ be a representative of $g$.

- If Pos $(u)+N e g(u)<k$, then $u$ is the unique geodesic representative of $g$.

- If Pos $(u)+N e g(u)=k$, then $u$ is geodesic, but $g$ has several geodesic representatives.

- If $\operatorname{Pos}(u)+N e g(u)>k$, then $u$ is not geodesic.

In the case $k=3$, Proposition 4.3 appears in [25, Theorem 1.1] (without the distinction between uniqueness and non-uniqueness of geodesics). Our method of proof differs perceptibly from the one in $[25]$.

Before proving Proposition 4.3, we explain how to choose a particular geodesic representative of an element when the second case of Proposition 4.3 occurs.

Set $I=\operatorname{Pos}(u)$ and $J=\operatorname{Neg}(u)$. If $(I, J)=(k, 0)$, a particular geodesic representative can be choosen using Proposition 2.2. The case $(I, J)=(0, k)$ is treated similarly. When $I \geqslant 1, J \geqslant 1$, we have:

Proposition 4.4. Let $g \in A_{k}$ and let $u \in \mathbb{F}$ be a representative of $g$. Set $I=\operatorname{Pos}(u)$ and $J=N e g(u)$. Assume that $I \geqslant 1, J \geqslant 1$, and $I+J=k$. Then there exists a unique geodesic representative $\widehat{u}$ of $g$ with the following property:

$$
\begin{aligned}
& \exists v^{(1)}, v^{(2)}, v^{(3)} \in \mathbb{F}, \\
& \exists v_{-} \in\left\{\operatorname{prod}\left(a^{-1}, b^{-1} ; J\right), \operatorname{prod}\left(b^{-1}, a^{-1} ; J\right)\right\}, \exists v_{+} \in\{\operatorname{prod}(a, b ; I), \operatorname{prod}(b, a ; I)\}
\end{aligned}
$$

such that

$$
\begin{gathered}
\widehat{u}=v^{(1)} \cdot v_{-} \cdot v^{(2)} \cdot v_{+} \cdot v^{(3)} \\
\left\{\begin{array}{l}
\operatorname{Pos}\left(v^{(1)}\right) \leqslant I-1 \\
\operatorname{Neg}\left(v^{(1)}\right) \leqslant J-1
\end{array}, \quad\left\{\begin{array} { l } 
{ \operatorname { P o s } ( v ^ { ( 2 ) } ) \leqslant I - 1 } \\
{ \operatorname { N e g } ( v ^ { ( 2 ) } ) \leqslant J }
\end{array} , \text { and } \quad \left\{\begin{array}{l}
\operatorname{Pos}\left(v^{(3)}\right) \leqslant I \\
\operatorname{Neg}\left(v^{(3)}\right) \leqslant J-1
\end{array} .\right.\right.\right.
\end{gathered}
$$

Proof of Proposition 4.3. Let $g \in A_{k}$, let $u \in \mathbb{F}$ be a representative of $g$ and $v=\operatorname{Brack}(u)$. Set $I=\operatorname{Pos}(u), J=\operatorname{Neg}(u)$. Write $v=v_{1} \cdot \ldots \cdot v_{l}$ with $v_{i} \in T$.

- The case $I+J>k$.

Since $I \leqslant k$ and $J \leqslant k$ (cf. the definition of $I$ and $J$ ), this implies that both $I$ and $J$ are positive. Pick $v_{i} \in T^{+}$such that $\left|v_{i}\right|_{S}=I$ and $v_{j} \in T^{-}$such that $\left|v_{j}\right|_{S}=J$. Assume for instance that $i<j$. We have:

$$
v_{j}=\Delta^{-1}\left(v_{j}^{-1}\right)^{*}, \quad\left|\left(v_{j}^{-1}\right)^{*}\right|_{S}=k-\left|v_{j}\right|_{S}=k-J,
$$

with the convention that $\left(v_{j}^{-1}\right)^{*}$ is the empty word when $v_{j}=\Delta^{-1}$. Now move $\Delta^{-1}$ along $v$ towards $v_{i}$ and do the transformation $\left[v_{i} \Delta^{-1} \rightarrow\left(v_{i}^{-1}\right)^{*}\right]$. This produces a new representative of $g$ denoted by $w$. We now prove that $|w|_{S}<|v|_{S}$. Transforming $v$ into $w$ changes the length of only two elements: $v_{i}$ and $v_{j}$. More precisely, these two elements change respectively to $\left(v_{i}^{-1}\right)^{*}$ and $\left(v_{j}^{-1}\right)^{*}$ whose respective lengths are: $k-\left|v_{i}^{-1}\right|_{S}=k-\left|v_{i}\right|_{S}=k-I$ and $k-J$. Therefore, one has

$$
\left|\left(v_{i}^{-1}\right)^{*}\right|_{S}+\left|\left(v_{j}^{-1}\right)^{*}\right|_{S}=k-I+k-J<I+J=\left|v_{i}\right|_{S}+\left|v_{j}\right|_{S} .
$$

We conclude that $u$ is not geodesic. 
- The case $I+J<k$.

In particular, we have $I<k$ and $J<k$. According to Lemma 4.1, we have, for all $i \in$ $\{1, \ldots, l-1\}$,

$$
\operatorname{Last}\left(v_{i}\right)=\operatorname{First}\left(v_{i+1}\right) \text {. }
$$

Suppose first that $J=0$. Then all the $v_{i}$ 's belong to $M^{+}$, which implies that $g$ belongs to the monoid $A_{k}^{+}$and that $u$ is geodesic, see (8). It also implies, compare (26) and (10), that $v_{1} \cdot \ldots \cdot v_{l}$ is actually the Garside normal form of $g$, which leads to the uniqueness of the geodesic representative of $g$.

Next, suppose that $I=0$. This case reduces to the previous one by considering $v^{-1}$ and observing that $S=S^{-1}$.

Last, suppose that $I \geqslant 1$ and $J \geqslant 1$. Denote by $\operatorname{Gars}(v)=v_{1}^{\prime} \cdot \ldots \cdot v_{l}^{\prime} \cdot \Delta^{\delta}$ the output of the GARSIDE algorithm, and recall that this word is precisely the Garside normal form of $g$.

By looking at the GARsIDE algorithm, one observes that $|\delta|$ is exactly the number of indices $i \in\{1, \ldots, l\}$ such that $v_{i} \in M^{-}$.

Now, starting from the Garside normal form $v_{1}^{\prime} \cdot \ldots \cdot v_{l}^{\prime} \cdot \Delta^{\delta}$, we execute the Suitable-SPREAD procedure defined in Section 2.2.

This procedure is exactly the inverse of what we have done just before, and precisely leads to $v$ that we started from. The reason is the following: if $v_{i} \in M^{-}$, then we have:

$$
\left|v_{i}^{\prime}\right|_{S}=\left|\left(v_{i}^{-1}\right)^{*}\right|_{S}=k-\left|v_{i}\right|_{S} \geqslant k-J>I .
$$

On the other hand, if $v_{i} \in M^{+}$, then $\left|v_{i}^{\prime}\right|_{S}=\left|v_{i}\right|_{S} \leqslant I$. We obtain that there are exactly $|\delta|$ indices $i \in\{1, \ldots, l\}$ such that $\left|v_{i}^{\prime}\right|_{S}>I$. So these indices are precisely the ones choosen in the "Pick $i$ " line of the Suitable-SPREAD algorithm. We conclude that the word $u$ is geodesic.

Let us now prove the uniqueness. Let $\widetilde{u} \in \mathbb{F}$ be another geodesic representative of $g$. Set $\widetilde{v}=\operatorname{Brack}(\widetilde{u}), \widetilde{I}=\operatorname{Pos}(\widetilde{u}), \widetilde{J}=\operatorname{Neg}(\widetilde{u})$. Since neither $g$ nor $g^{-1}$ belong to $A_{k}^{+}$(recall that $I \geqslant 1$ and $J \geqslant 1$ ), we have $\widetilde{I} \geqslant 1$ and $\widetilde{J} \geqslant 1$. Also since $\widetilde{u}$ is geodesic, we have $\widetilde{I}+\widetilde{J} \leqslant k$ (first part of the proof).

In particular, $\widetilde{I}<k$ and $\widetilde{J}<k$, so we can apply the same argument as above. The Garside procedure applied to the input $\widetilde{v}$ followed by the SUITABLE-SPREAD procedure leads back to $\widetilde{v}$. Since $\operatorname{Gars}(v)=\operatorname{Gars}(\widetilde{v})$ (uniqueness of the Garside normal form), we conclude that $\widetilde{v}=v$ and $\widetilde{u}=u$.

- The case $I+J=k$.

Suppose first that $J=0$. Then all the $v_{i}$ 's belong to $T^{+}$with some of them equal to $\Delta$. Again, according to (8), the word $u$ is geodesic. The non-uniqueness is directly deduced from the fact that $\Delta=\operatorname{prod}(a, b ; k)=\operatorname{prod}(b, a ; k)$. The case $I=0$ again reduces to the latter one.

Now, suppose that $I \geqslant 1$ and $J \geqslant 1$. Since $I+J=k$, we have $I<k$ and $J<k$, therefore we can apply the Garside algorithm. Set $\operatorname{Gars}(v)=v_{1}^{\prime} \cdot \ldots \cdot v_{l}^{\prime} \cdot \Delta^{\delta}$, where $|\delta|=\operatorname{Card}\left\{i \mid v_{i}^{\prime} \in M^{-}\right\}$.

We introduce the following sets of indices:

$$
\begin{aligned}
& A=\left\{\left.i\left|v_{i} \in M^{+},\right| v_{i}\right|_{S}=I\right\}=\left\{\left.i\left|v_{i}^{\prime} \in M^{+},\right| v_{i}^{\prime}\right|_{S}=I\right\} \\
& B=\left\{\left.i\left|v_{i} \in M^{-},\right| v_{i}\right|_{S}=J\right\}=\left\{\left.i\left|v_{i}^{\prime} \in M^{+},\right| v_{i}^{\prime}\right|_{S}=I\right\} \\
& C=\left\{\left.i\left|v_{i} \in M^{-},\right| v_{i}\right|_{S}<J\right\}=\left\{\left.i\left|v_{i}^{\prime} \in M^{+},\right| v_{i}^{\prime}\right|_{S}>I\right\}
\end{aligned}
$$


Observe that $A$ and $B$ are both non-empty. Apply now the SuITABLE-SPREAD algorithm to the Garside normal form $v_{1}^{\prime} \cdot \ldots \cdot v_{l}^{\prime} \cdot \Delta^{\delta}$ of $g$. If one chooses the set $B \cup C$ in the "Pick $i$ " line of the algorithm (that is, if $I=\{1, \ldots, l\} \backslash(B \cup C)$ at the end of the while loop), then the output is precisely $v$. Therefore $v$ is geodesic according to Proposition 2.3. If one replaces some of the indices from $B$ by indices from $A$ in the "Pick $i$ " line, then the output is another geodesic representative of $g$.

Proof of Proposition 4.4. Let $g \in A_{k}$, let $u \in \mathbb{F}$ be a representative of $g$ and $v=\operatorname{Brack}(u)$. Set $I=\operatorname{Pos}(u), J=\operatorname{Neg}(u)$, and assume that $I \geqslant 1, J \geqslant 1$, and $I+J=k$. Write $v=v_{1} \cdot \ldots \cdot v_{l}$ with $v_{i} \in M$. Run the GARside procedure on the input $v$, and denote the output by $v_{1}^{\prime} \cdot \ldots \cdot v_{l}^{\prime} \cdot \Delta^{\delta}$, with $|\delta|=\operatorname{Card}\left\{i \mid v_{i}^{\prime} \in M^{-}\right\}$.

In order to prove the existence and the uniqueness stated in Proposition 4.4, the central point is the following: the requested geodesic representative $\widehat{u}$ of $g$ satisfying (24) and (25) is exactly the output of the SUITABLE-SPREAD procedure applied to $v_{1}^{\prime} \cdot \ldots \cdot v_{l}^{\prime} \cdot \Delta^{\delta}$ if, in the "Pick $i$ " line of the algorithm, among all the elements $v_{i}^{\prime}$ of maximal length, one chooses the $|\delta|$ smallest possible indices. We call this the left-greedy choice.

We now give some details.

\section{- Existence.}

Denote by $w=w_{1} \cdot \ldots \cdot w_{l}$ the output of the SUITABLE-SPREAD algorithm when the left-greedy choice is performed. Recall that $S^{+}=\{a, b\}$ and set $S^{-}=\left\{a^{-1}, b^{-1}\right\}$.

We denote by $\alpha$ the index defined by

$$
\alpha=1+\max \left\{i \mid \operatorname{Pos}\left(w_{1} \cdot \ldots \cdot w_{i}\right) \leq I-1, \quad \operatorname{Neg}\left(w_{1} \cdot \ldots \cdot w_{i}\right) \leq J-1\right\},
$$

and $\alpha=1$ if the set of indices $i$ is empty. We set $v^{(1)}=w_{1} \cdot \ldots \cdot w_{\alpha-1}\left(v^{(1)}\right.$ may be the empty word).

We assert that $w_{\alpha} \in M^{-}$and $\left|w_{\alpha}\right|_{S^{-}}=J$. Let us prove this point. Suppose that $w_{\alpha} \in M^{+}$ and $\left|w_{\alpha}\right|_{S^{+}}=I$. Then, there exists $\beta>\alpha$ such that $w_{\beta} \in M^{-}$and $\left|w_{\beta}\right|_{S^{-}}=J$. Write $w_{\beta}=$ $\Delta^{-1}\left(w_{\beta}^{-1}\right)^{*}$, move $\Delta^{-1}$ to the left towards $w_{\alpha}$, and do the transformation $\left[w_{\alpha} \Delta^{-1} \rightarrow\left(w_{\alpha}^{-1}\right)^{*}\right]$. This exhibits a contradiction with the fact that the SUITABLE-SPREAD procedure was run in the left-greedy way: the index $\alpha$ should have been picked instead of the index $\beta$.

Set $v_{-}=w_{\alpha}$. Denote by $\beta$ the index defined by

$$
\beta=1+\max \left\{i \geqslant \alpha+1 \mid \operatorname{Pos}\left(w_{\alpha+1} \cdot \ldots \cdot w_{i}\right) \leq I-1, \quad \operatorname{Neg}\left(w_{\alpha+1} \cdot \ldots \cdot w_{i}\right) \leq J\right\},
$$

and $\beta=1$ if the set of indices $i$ is empty. Set $v^{(2)}=w_{\alpha+1} \cdot \ldots \cdot w_{\beta-1}$ (again, this may be the empty word). Clearly, $w_{\beta} \in M^{+}$and $\left|w_{\beta}\right|_{S^{+}}=I$. We set $v_{+}=w_{\beta}$. What remains to be proved is the following: $\operatorname{Neg}\left(w_{\beta+1} \cdot \ldots \cdot w_{l}\right) \leq J-1$. If it were false, there would exist $\gamma \geqslant \beta+1$ such that $w_{\gamma} \in M^{-}$and $\left|w_{\gamma}\right|_{S^{-}}=J$. Repeating the above argument with respectively $w_{\beta}$ and $w_{\gamma}$ instead of $w_{\alpha}$ and $w_{\beta}$ leads again to a contradiction.

Finally, we set $v^{(3)}=w_{\beta+1} \cdot \ldots \cdot w_{l}$. One has $w=v^{(1)} \cdot v_{-} \cdot v^{(2)} \cdot v_{+} \cdot v^{(3)}$ and the word $\widehat{u}=w$ is as required.

- Uniqueness.

Suppose that $\widehat{u}$ is a geodesic representative of $g$ such that $\widehat{u}=v^{(1)} \cdot v_{-} \cdot v^{(2)} \cdot v_{+} \cdot v^{(3)}$ satisfies (24) and (25). Write $v^{(1)}=w_{1} \cdot \ldots \cdot w_{\alpha-1}, v_{-}=w_{\alpha}, v^{(2)}=w_{\alpha+1} \cdot \ldots \cdot w_{\beta-1}, v_{+}=w_{\beta}$, and $v^{(3)}=w_{\beta+1} \cdot \ldots \cdot w_{l}$ with $w_{i} \in M$. 
Executing the GARSIDE procedure with input $w_{1} \cdot \ldots \cdot w_{l}$ provides the Garside normal form $v_{1}^{\prime} \cdot \ldots \cdot v_{l}^{\prime} \cdot \Delta^{\delta}$ of $g$ as output. The indices $\alpha$ and $\beta$ are related to this Garside normal form by means of the following formulas:

$$
\begin{gathered}
\alpha=1+\max \left\{i \mid \operatorname{Pos}\left(v_{1}^{\prime} \cdot \ldots \cdot v_{i}^{\prime}\right) \neq I\right\} \\
\beta=1+\max \left\{i\left|\operatorname{Card}\left\{\left.j|j \leqslant i,| v_{j}^{\prime}\right|_{S} \geqslant I\right\}=\right| \delta \mid\right\} .
\end{gathered}
$$

Therefore, $\alpha$ and $\beta$ are unique. Another consequence of these formulas is that $\widehat{u}$ is exactly the output of the SUITABLE-SPREAD procedure when the left-greedy choice is performed.

A close inspection of the proof of Proposition 4.3 also provides the following converse to Proposition 2.3:

Lemma 4.5. Let $g \in A_{k}$ and let $v$ be the Garside normal form of $g$. Any geodesic representative of $g$ with respect to $S$ can be obtained as an output to the SUITABLE-SPREAD algorithm with input $v$.

Application to translation lengths. Another by-product of Proposition 4.3 is to enable the computation of translation lengths. Let $G$ be a group generated by the finite set $S$. The translation length (or translation number) of an element $g \in G$ with respect to $S$ is

$$
\tau_{S}(g)=\lim _{n \rightarrow+\infty} \frac{\left|g^{n}\right|_{S}}{n} .
$$

The limit exists by subadditivity. It is known that for any word-hyperbolic group $G$ and for any finite generating set $S$ of $G$, the length $\tau_{S}(g)$ is a rational number for every $g \in G$ (see [1]). If $G$ is a Garside group and $S$ the set of simple elements, then the set of all the translation lengths $\left\{\tau_{S}(g), g \in G\right\}$ is a discrete set (see [20]). In the case of Artin groups of dihedral type, we have:

Proposition 4.6. For $S=\left\{a, b, a^{-1}, b^{-1}\right\}$ and $g \in A_{k}$, the translation length $\tau_{S}(g)$ is an integer. More precisely, $\tau_{S}(g)=\left|g^{\prime}\right|_{S}$ where $g^{\prime}$ is an element of shortest length in $[g]$, the conjugacy class of $g$ in $G$.

In the case $A_{3}=B_{3}$, this statement is due to Sabalka (see [25]). Using Proposition 4.3, the proof of [25] can be directly adapted to get Proposition 4.6.

\section{Computing the growth series}

Define the sets:

$$
\mathcal{S}_{I, J}=\{u \in \mathbb{E} \mid \operatorname{Pos}(u)=I, \operatorname{Neg}(u)=J\} .
$$

Let $S_{I, J} \in \mathbb{N}[[x]]$ be the generating series of $\mathcal{S}_{I, J}$, that is:

$$
S_{I, J}=\sum_{u \in \mathcal{S}_{I, J}} x^{|u|_{S}}=\sum_{n \in \mathbb{N}} \#\left\{\left.u \in \mathcal{S}_{I, J}|| u\right|_{S}=n\right\} x^{n} .
$$

According to Proposition 4.3, the sets $\mathcal{S}_{I, J}, I+J \leqslant k$, partition the set of all the geodesic representatives of $A_{k}$. Hence we have:

$$
\mathcal{G}\left(A_{k}, S\right)=\sum_{I+J \leqslant k} S_{I, J}
$$


Now we turn our attention to the spherical growth series. According to Proposition 4.3, the sets $\mathcal{S}_{I, J}, I+J=k$, contain several geodesic representatives of the same element. The sets $\widehat{\mathcal{S}}_{I, J}$, $I+J=k$, to be defined soon, will be cross-sections of the sets $\mathcal{S}_{I, J}, I+J=k$.

Consider $\mathcal{S}_{k, 0}$. We denote by $\widehat{\mathcal{S}}_{k, 0}$ the subset of $\mathcal{S}_{k, 0}$ consisting of the words which are in Garside normal form, see Lemma 2.2. More precisely:

$$
\widehat{\mathcal{S}}_{k, 0}=\left\{u \in \mathbb{F} \mid u=u_{1} \cdots u_{n} \Delta^{\delta}, \delta \geqslant 1, \forall i, u_{i} \in M^{+}, \operatorname{Last}\left(u_{i}\right)=\operatorname{First}\left(u_{i+1}\right)\right\} .
$$

We define $\widehat{\mathcal{S}}_{0, k}$ similarly over the subalphabet $\left\{a^{-1}, b^{-1}\right\}$.

For $I+J=k, I \geqslant 1, J \geqslant 1$, let $\widehat{\mathcal{S}}_{I, J}$ be the subset of $\mathcal{S}_{I, J}$ defined by:

$$
\widehat{\mathcal{S}}_{I, J}=\{\widehat{u} \in \mathbb{E} \mid(24) \text { and (25) hold }\} .
$$

Denote by $\widehat{S}_{I, J} \in \mathbb{N}[[x]], I+J=k$, the corresponding generating series. According to Propositions 4.3 and 4.4, the sets $\mathcal{S}_{I, J}, I+J \leqslant k-1$, and $\widehat{\mathcal{S}}_{I, J}, I+J=k$, are disjoint and their union forms a geodesic cross-section of $A_{k}$. Consequently, the spherical growth series of $A_{k}$ is:

$$
\mathcal{S}\left(A_{k}, S\right)=\sum_{I+J \leqslant k-1} S_{I, J}+\sum_{\substack{I \geqslant 1, J \geqslant 1 \\ I+J=k}} \widehat{S}_{I, J}+\widehat{S}_{k, 0}+\widehat{S}_{0, k} .
$$

In order to compute the series $\mathcal{G}\left(A_{k}, S\right)$ and $\mathcal{S}\left(A_{k}, S\right)$, we have to partition the languages $\mathcal{S}_{i, j}$ (or $\widehat{\mathcal{S}}_{i, j}$ ) in a certain way, which is illustrated in Figure 1.
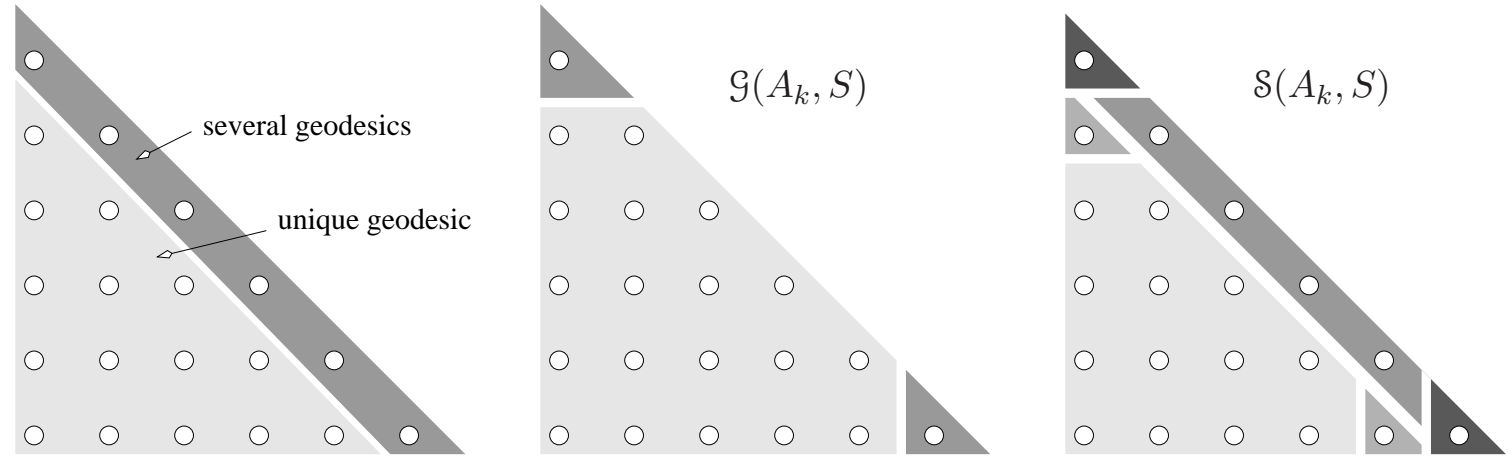

Figure 1: Snapshot of the proof of Theorem 3.1.

Let us build automata recognizing the languages $\mathcal{S}_{I, J}$ and $\widehat{\mathcal{S}}_{I, J}$.

According to (8), we have $\cup_{i \leqslant k} \mathcal{S}_{i, 0}=\{a, b\}^{*}$. Similarly, we have $\cup_{j \leqslant k} \mathcal{S}_{0, j}=\left\{a^{-1}, b^{-1}\right\}^{*}$. It follows that:

$$
\sum_{i \leqslant k} S_{i, 0}=\sum_{j \leqslant k} S_{0, j}=\frac{1}{1-2 x}
$$

Consider now $I, J$, such that $I+J \leqslant k$ and $(I, J) \notin\{(k, 0),(0, k)\}$. Consider the language $\cup_{i \leqslant I, j \leqslant J} \mathcal{S}_{i, j}$. It is recognized by the deterministic automaton $\mathbf{A}_{\leqslant I, \leqslant J}$ defined as follows:

- States: $1 \cup \Sigma_{I}^{+} \cup \Sigma_{J}^{-}$; initial state: 1; final states: $1 \cup \Sigma_{I}^{+} \cup \Sigma_{J}^{-}$;

- Transitions: $\forall v \in \Sigma_{I}^{+} \cup \Sigma_{J}^{-}, 1 \stackrel{v}{\longrightarrow} v$, and $\forall u, v \in \Sigma_{I}^{+} \cup \Sigma_{J}^{-}, u \stackrel{v}{\longrightarrow} v$ if $\operatorname{Last}(u)=\operatorname{First}(v)$. 
The proof follows directly from Lemma 4.1. Now let us translate this at the level of the generating series. Recall that $X_{0}=0$ and for $n \geqslant 1, X_{n}=x+x^{2}+\cdots+x^{n}$.

Lemma 5.1. We have:

$$
\forall I, J, I+J \leqslant k,(I, J) \neq(k, 0),(0, k), \quad \sum_{i \leqslant I, j \leqslant J} S_{i, j}=\frac{1+X_{I}+X_{J}}{1-X_{I}-X_{J}} .
$$

Consequently, we have:

$$
S_{k, 0}=S_{0, k}=\frac{1}{1-2 x}-\frac{1+X_{k-1}}{1-X_{k-1}}, \quad \forall I \leqslant k-1, S_{I, 0}=S_{0, I}=\frac{1+X_{I}}{1-X_{I}}-\frac{1+X_{I-1}}{1-X_{I-1}},
$$

and for all $I, J, I \geqslant 1, J \geqslant 1, I+J \leqslant k$,

$$
\begin{aligned}
S_{I, J} & =\sum_{i \leqslant I, j \leqslant J} S_{i, j}-\sum_{i \leqslant I-1, j \leqslant J} S_{i, j}-\sum_{i \leqslant I, j \leqslant J-1} S_{i, j}+\sum_{i \leqslant I-1, j \leqslant J-1} S_{i, j} \\
& =\frac{1+X_{I}+X_{J}}{1-X_{I}-X_{J}}-\frac{1+X_{I-1}+X_{J}}{1-X_{I-1}-X_{J}}-\frac{1+X_{I}+X_{J-1}}{1-X_{I}-X_{J-1}}+\frac{1+X_{I-1}+X_{J-1}}{1-X_{I-1}-X_{J-1}} .
\end{aligned}
$$

Proof. Assume that $(I, J) \neq(k, 0),(0, k)$. Consider $u \in \cup_{i \leqslant I, j \leqslant J} \mathcal{S}_{i, j}$ and $v=$ $v_{1} \cdots v_{n}=\operatorname{Brack}(u)$. According to Lemma 4.1, we have: $v_{1}$ may be any element of $\Sigma_{I}^{+} \sqcup \Sigma_{J}^{-}$; if $v_{i}=w_{i} a$ then $v_{i+1}$ may be any element of $\{a, a b, \ldots, \operatorname{prod}(a, b ; I)\} \sqcup$ $\left\{b^{-1}, b^{-1} a^{-1}, \ldots, \operatorname{prod}\left(b^{-1}, a^{-1} ; J\right)\right\} ; \quad$ if $v_{i}=w_{i} a^{-1}$ then $v_{i+1}$ may be any element of $\{b, b a, \ldots, \operatorname{prod}(b, a ; I)\} \sqcup\left\{a^{-1}, a^{-1} b^{-1}, \ldots, \operatorname{prod}\left(a^{-1}, b^{-1} ; J\right)\right\}$; and so on. At the level of the generating series, we get:

$$
\sum_{i \leqslant I, j \leqslant J} S_{i, j}=1+\frac{2 x+\cdots+2 x^{I}+2 x+\cdots+2 x^{J}}{1-x-\cdots-x^{I}-x-\cdots-x^{J}}=\frac{1+X_{I}+X_{J}}{1-X_{I}-X_{J}} .
$$

The other equalities follow easily.

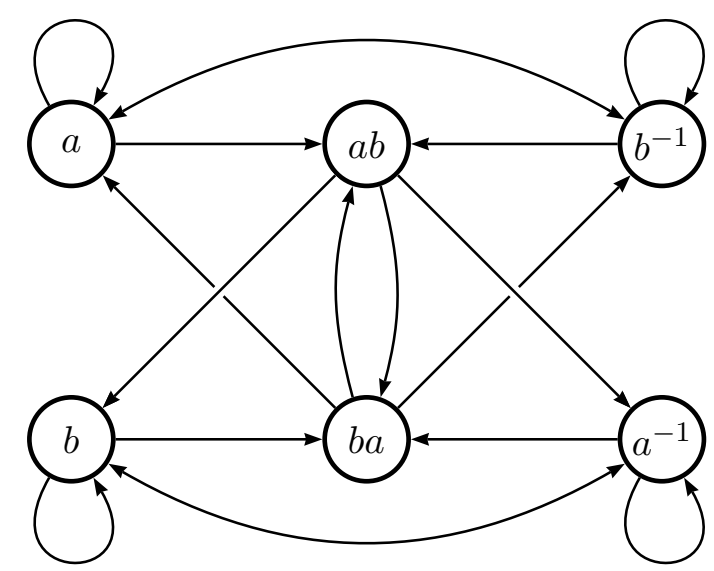

Figure 2: The graph $\mathfrak{A}_{\leqslant 2, \leqslant 1}$.

Denote by $\mathfrak{A}_{\leqslant I, \leqslant J}$ the labelled directed graph obtained from the automaton $\mathbf{A}_{\leqslant I, \leqslant J}$ by removing the state 1 and the outgoing transitions from 1 (in $\mathfrak{A}_{\leqslant I, \leqslant J}$, no initial or final state is specified). The graph $\mathfrak{A}_{\leqslant 2, \leqslant 1}$ is represented in Figure 2 (we have omitted the labels on the arcs). 
Let $\mathfrak{B}_{I, 0}$ be the graph consisting of two isolated nodes labelled respectively by $\operatorname{prod}(a, b ; I)$ and $\operatorname{prod}(b, a ; I)$. Let $\mathfrak{B}_{0, J}$ be the graph consisting of two isolated nodes labelled respectively by $\operatorname{prod}\left(a^{-1}, b^{-1} ; J\right)$ and $\operatorname{prod}\left(b^{-1}, a^{-1} ; J\right)$.

For $I \leqslant k-1$, a direct argument shows that the language $\mathcal{S}_{I, 0}$ is recognized by the deterministic automaton whose structure is given in Figure 3.

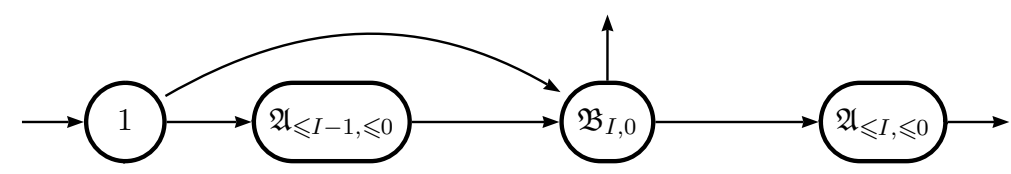

Figure 3: Automaton recognizing $\mathcal{S}_{I, 0}$.

The initial state is the state 1 . The final states are all the states from the two right-most blocks: $\mathfrak{B}_{I, 0}$ and $\mathfrak{A}_{\leqslant I, \leqslant 0}$. Between the initial state and the outgoing blocks, the transitions are: $1 \stackrel{v}{\rightarrow} v$. Between the other blocks, the transitions are: $u \stackrel{v}{\rightarrow} v$ if Last $(u)=\operatorname{First}(v)$.

Consider the language $\mathcal{S}_{I, J}, I \geqslant 1, J \geqslant 1, I+J \leq k$. Similarly, a straightforward argument shows that it is recognized by the deterministic automaton whose structure is given in Figure 4 .

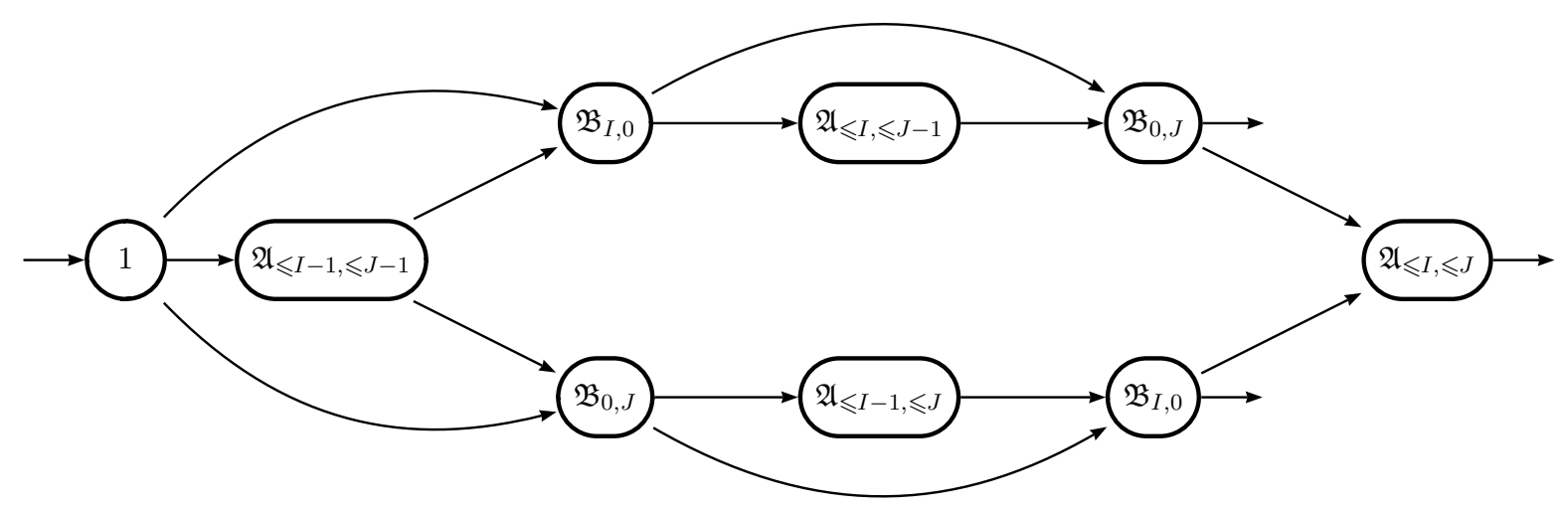

Figure 4: Automaton recognizing $\mathcal{S}_{I, J}$.

Consider the language $\widehat{\mathcal{S}}_{k, 0}$ (the case of $\widehat{\mathcal{S}}_{0, k}$ is treated similarly). By definition, see (29), it is recognized by the deterministic automaton whose structure is given in Figure 5.

Consider now the language $\widehat{\mathcal{S}}_{I, J}, I+J=k, I \geqslant 1, J \geqslant 1$. A direct transposition of the conditions (24) and (25) yields the following. The language $\widehat{\mathcal{S}}_{I, J}$ is recognized by the deterministic automaton whose structure is given in Figure 6.

When translating the above on the generating series, we get:

Lemma 5.2. We have:

$$
\widehat{S}_{k, 0}=\widehat{S}_{0, k}=\frac{1+X_{k-1}}{1-X_{k-1}} \frac{x^{k}}{1-x^{k}}
$$




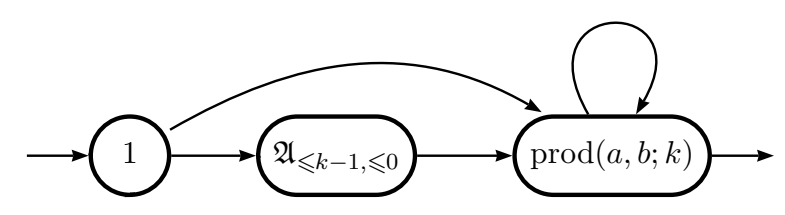

Figure 5: Automaton recognizing $\widehat{\mathcal{S}}_{k, 0}$.

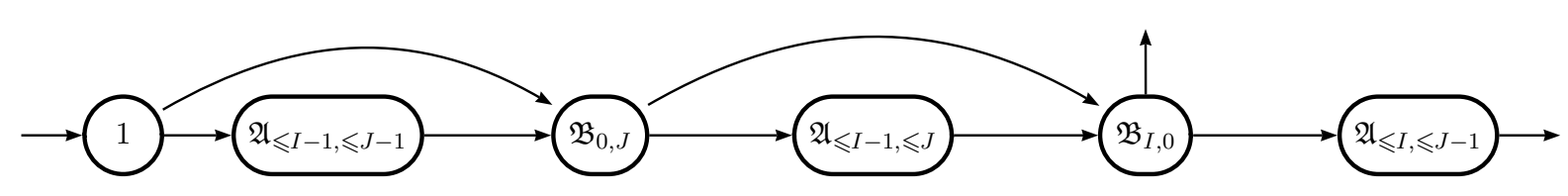

Figure 6: Automaton recognizing $\widehat{\mathcal{S}}_{I, J}$.

Consider $I, J$ with $I \geqslant 1, J \geqslant 1, I+J=k$. We have:

$$
\widehat{S}_{I, J}=\frac{2 x^{k}}{\left(1-X_{I-1}-X_{J-1}\right)\left(1-X_{I-1}-X_{J}\right)\left(1-X_{I}-X_{J-1}\right)} .
$$

Using (28) and Lemma 5.1, we get a rational expression for $\mathcal{G}\left(A_{k}, S\right)$. Using (31) and Lemmas 5.1 and 5.2 , we get a rational expression for $\mathcal{S}\left(A_{k}, S\right)$. To get the simple formulas in Theorem 3.1 , we need an additional trick.

Lemma 5.3. We have, for $\ell \leqslant k$,

$$
\sum_{I+J \leqslant \ell} S_{I, J}=S_{0, \ell}+S_{\ell, 0}+\sum_{\substack{I, J \geqslant 1 \\ I+J=\ell}} \sum_{\substack{0 \leqslant i \leqslant I \\ 0 \leqslant j \leqslant J}} S_{i, j}-\sum_{\substack{I, J \geqslant 1 \\ I+J=\ell-1}} \sum_{\substack{0 \leqslant i \leqslant I \\ 0 \leqslant j \leqslant J}} S_{i, j} .
$$

Proof. This is shown by a simple counting argument. (Here, the specific values of the series $S_{i, j}$ do not play any role.)

\section{Proof of Theorem 3.1.}

Consider (28) and replace the right-hand side using (37) for $\ell=k$. We get:

$$
\mathcal{G}\left(A_{k}, S\right)=S_{k, 0}+S_{0, k}+\sum_{\substack{I, J \geqslant 1 \\ I+J=k}} \sum_{\substack{0 \leqslant i \leqslant I \\ 0 \leqslant j \leqslant J}} S_{i, j}-\sum_{\substack{I, J \geqslant 1 \\ I+J=k-1}} \sum_{\substack{0 \leqslant i \leqslant I \\ 0 \leqslant j \leqslant J}} S_{i, j} .
$$

Replace the various terms on the right-hand side using (33) and (34). We get precisely (15) in Theorem 3.1.

Consider (31) and replace the first sum in the right-hand side using (37) for $\ell=k-1$. We get:

$$
\begin{gathered}
\mathcal{S}\left(A_{k}, S\right)=S_{k-1,0}+S_{0, k-1}+\sum_{\substack{I, J \geqslant 1 \\
I+J=k-1}} \sum_{\substack{0 \leqslant i \leqslant I \\
0 \leqslant j \leqslant J}} S_{i, j}-\sum_{\substack{I, J \geqslant 1 \\
I+J=k-2}} \sum_{\substack{0 \leqslant i \leqslant I \\
0 \leqslant j \leqslant J}} S_{i, j} \\
+\sum_{\substack{I, J \geqslant 1 \\
I+J=k}} \widehat{S}_{I, J}+\widehat{S}_{k, 0}+\widehat{S}_{0, k} .
\end{gathered}
$$


Now replace the various terms on the right-hand side using (33), (34), (35), and (36). We obtain (16) in Theorem 3.1.

\section{References}

[1] G. Baumslag, S.M. Gersten, M. Shapiro, and H. Short. Automatic groups and amalgams. J. Pure Appl. Algebra, 76(3):229-316, 1991.

[2] M. Berger. Minimum crossing numbers for 3-braids. J. Phys. A, 27(18):6205-6213, 1994.

[3] D. Bessis. The dual braid monoid. Ann. Sci. École Norm. Sup. (4), 36(5):647-683, 2003.

[4] J. Birman, K.-H. Ko, and S.-J. Lee. A new approach to the word and conjugacy problems in the braid groups. Adv. Math., 139(2):322-353, 1998.

[5] M. Brazil. Monoid growth functions for braid groups. Internat. J. Algebra Comput., 1(2):201-205, 1991.

[6] S. Burckel. Syntactical methods for braids of three strands. J. Symbolic Comput., 31(5):557-564, 2001.

[7] J. Cannon. The combinatorial structure of cocompact discrete hyperbolic groups. Geom. Dedicata, 16(2):123-148, 1984.

[8] R. Charney. Artin groups of finite type are biautomatic. Math. Ann., 292(4):671-684, 1992.

[9] R. Charney. Geodesic automation and growth functions for Artin groups of finite type. Math. Ann., 301(2):307-324, 1995.

[10] R. Charney and J. Meier. The language of geodesics for Garside groups. Math. Z. 248(3):495-509, 2004.

[11] P. Dehornoy. Groupes de Garside. Ann. Sci. École Norm. Sup. (4), 35(2):267-306, 2002.

[12] P. Deligne. Les immeubles des groupes de tresses généralisés. Invent. Math., 17:273-302, 1972.

[13] D. Epstein, J. Cannon, D. Holt, S. Levy, M. Paterson, and W. Thurston. Word processing in groups. Jones and Bartlett, Boston, 1992.

[14] D. Epstein, D. Holt, and S. Rees. The use of Knuth-Bendix methods to solve the word problem in automatic groups. J. Symbolic Comput., 12(4-5):397-414, 1991.

[15] F. Garside. The braid groups and other groups. Quart. J. Math. Oxford, 20:235-254, 1969.

[16] É. Ghys and P. de la Harpe, editors. Sur les groupes hyperboliques d'après Mikhael Gromov, volume 83 of Progress in Mathematics. Birkhäuser, Boston, 1990.

[17] R. Grigorchuk and P. de la Harpe. On problems related to growth, entropy, and spectrum in group theory. J. Dynam. Control Systems, 3(1):51-89, 1997.

[18] M. Gromov. Hyperbolic groups. In Essays in group theory, volume 8 of Math. Sci. Res. Inst. Publ., pages 75-263. Springer, 1987.

[19] D. Krammer. Braid groups are linear. Ann. of Math. (2), 155(1):131-156, 2002. 
[20] S.J. Lee. Growth of minimal word-length in Garside groups. oai:arXiv.org: math.GT/0411470v1, 2004.

[21] J. Loeffler, J. Meier, and J. Worthington. Graph products and Cannon pairs. Internat. J. Algebra Comput., 12(6):747-754, 2002.

[22] J. Michel. A note on words in braid monoids. J. Algebra, 215(1):366-377, 1999.

[23] P. Papasoglu. Strongly geodesically automatic groups are hyperbolic. Invent. Math., 121(2):323-334, 1995.

[24] M. Picantin. Petits groupes gaussiens. PhD thesis, Univ. Caen, 2000.

[25] L. Sabalka. Geodesics in the braid group on three strands. In Group theory, statistics, and cryptography, volume 360 of Contemp. Math., pages 133-150. Amer. Math. Soc., 2004.

[26] M. Stoll. Rational and transcendental growth series for the higher Heisenberg groups. Invent. Math., 126(1):85-109, 1996.

[27] P. Xu. The genus of closed 3-braids. J. Knot Theory Ramifications, 1(3):303-326, 1992.

[28] P. Xu. Growth of the positive braid semigroups. J. Pure Appl. Algebra, 80(2):197-215, 1992. 